TRANSACTIONS OF THE

AMERICAN MATHEMATICAL SOCIETY

Volume 356, Number 5, Pages 2067-2079

S 0002-9947(03)03372-5

Article electronically published on November 12, 2003

\title{
GROMOV TRANSLATION ALGEBRAS OVER DISCRETE TREES ARE EXCHANGE RINGS
}

\author{
P. ARA, K. C. O'MEARA, AND F. PERERA
}

\begin{abstract}
It is shown that the Gromov translation ring of a discrete tree over a von Neumann regular ring is an exchange ring. This provides a new source of exchange rings, including, for example, the algebras $G(0)$ of $\omega \times \omega$ matrices (over a field) of constant bandwidth. An extension of these ideas shows that for all real numbers $r$ in the unit interval [0,1], the growth algebras $G(r)$ (introduced by Hannah and O'Meara in 1993) are exchange rings. Consequently, over a countable field, countable-dimensional exchange algebras can take any prescribed bandwidth dimension $r$ in $[0,1]$.
\end{abstract}

\section{INTRODUCTION}

This paper introduces a new and large source of exchange rings, in the form of certain Gromov translation rings, which potentially could aid the development of the theory of exchange rings as well as pointing to future directions of interest to workers in "coarse" geometry. Gromov introduced translation algebras (over the real or complex fields) in the early 1990's as certain algebras of infinite matrices, whose entries are indexed by a given "discrete" metric space $X$, and which encode "coarse" geometric information about $X$. (See $\S 1$ for the precise definitions.) To date, translation algebras do not appear to have been studied much by ring theorists. However, in a recent paper [5], the authors used translation algebras over a general field to tackle an old problem of Kaplansky's on group algebras in characteristic $p$.

Exchange rings were introduced in the early 1970's by Warfield because of the nice "exchange property" for their finitely generated projective modules. Following a later Goodearl and Nicholson elementwise characterization (via idempotents) of these rings, it became clear that exchange rings also provide a natural generalization of (von Neumann) regular rings. And indeed, in the late 1990's exchange rings have provided a common setting for a number of new and important results for both regular rings and various operator algebras $\left(C^{*}\right.$-algebras of real rank zero). Central to this is a common bond shared by exchange rings and regular rings; namely, direct

Received by the editors September 27, 2002 and, in revised form, April 15, 2003.

2000 Mathematics Subject Classification. Primary 16E50, 16D70, 16S50.

Key words and phrases. Translation algebra, exchange ring, von Neumann regular ring, infinite matrices, bandwidth dimension.

The first and third authors were partially supported by DGESIC, and by the Comissionat per Universitats i Recerca de la Generalitat de Catalunya. The initial ideas for this paper were discussed while the second author was visiting the Centre de Recerca Matemàtica, Institut d'Estudis Catalans in Barcelona, and he thanks this institution for its support and hospitality. 
sums of their finitely generated projective modules have the common refinement property. See [2, 3, 4, 15, 16] for some details and further references.

Although examples and constructions of exchange rings abound, there is a pressing need for new constructions to aid the development of the theory. For example, the Fundamental Separativity Problem for exchange rings ("Is every exchange ring separative?") is still open, despite a strong consensus that non-separative exchange rings should exist. See $3,4,7$ for more background on this problem.

Our principal result (Theorem 2.7) concerns the translation ring $T(X, R)$ of a "discrete" metric space $X$ over a general ring $R$. We show that $T(X, R)$ is an exchange ring for any discrete tree $X$ (endowed with the path metric) and any regular ring $R$. One immediate corollary (taking $X=\mathbb{N}$ with the Euclidean metric) is that the ring of all $\omega \times \omega$ matrices (over a regular ring $R$ ) that have constant bandwidth is an exchange ring. This appears to be new even in the case where $R$ is a field. In that setting, the papers 10, 11, 13, referred to the corresponding algebra of constant bandwidth matrices as the growth algebra $G(0)$, because it was the first of a whole spectrum of growth algebras $G(r)$, for $r$ in the unit interval $[0,1]$, that were used to define the bandwidth dimension of an arbitrary countable-dimensional algebra. For $0<r \leq 1$, the $G(r)$ are still translation algebras relative to a suitable pseudo-metric, and an easy extension (Theorem 3.1) of our main result shows that all the $G(r)$ are exchange algebras. (In fact, this holds also when the matrix entries are from any regular ring.) An interesting consequence of this is that, over a countable field, there exists a countable-dimensional exchange algebra of any prescribed bandwidth dimension $r \in[0,1]$.

We would like to thank John Roe for bringing translation algebras to the attention of the second author following the publication of [10, 11, 13].

\section{Preliminaries}

Let $R$ be a (unital) ring and let $(X, d)$ be a "locally finite" metric space, in the sense that all balls of finite radius are finite. We also implicitly assume $X$ is infinite, whence countably infinite. (In 5] we simply referred to these spaces as "discrete" metric spaces, but that term has a number of other interpretations.) Following Gromov [9, p. 262], we define the translation ring $T(X, R)$ of $X$ over $R$ to be the ring of all square matrices $(a(x, y))$, indexed by $X \times X$ and with entries from $R$, such that $a(x, y)=0$ whenever $d(x, y)>\ell$ for some constant $\ell$ depending on the matrix. The least such $\ell$ is called the bandwidth of the matrix. The translation ring also makes sense when $d$ is just a (locally finite) pseudo-metric, meaning that $d(x, y)=0$ does not necessitate $x=y$. We will need this broader setting in Section [3, The simplest example of a translation ring occurs when $X=\mathbb{N}$ has the Euclidean metric and $R=F$ is a field. Then $T(\mathbb{N}, F)$ is just the algebra of all $\omega \times \omega$ matrices over $F$ with constant bandwidth in the classical sense, i.e. of the form:

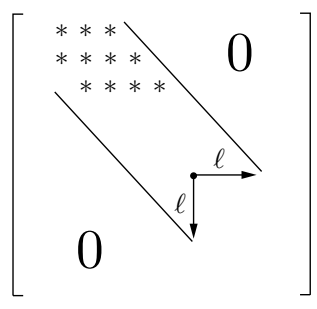


An important class of translation rings arises from discrete connected graphs $\Gamma$, in particular discrete trees (here "discrete" means having only finitely many edges at each vertex, although we implicitly assume that there are infinitely many vertices): let $X$ be the vertex set of $\Gamma$ and take $d(x, y)$ as the minimum of the lengths of the paths joining $x$ and $y$. One interesting subclass of these translation rings comes from taking $\Gamma$ as the Cayley graph of a finitely generated group $G$ with respect to a finite generating set $S$. The metric $d$ in this case is just the word metric in $S$ on $X=G$. See [5] for a recent application of translation rings in this setting.

The growth algebras $G(r)$, introduced by Hannah and O'Meara [10, 11, 13] in the early 1990's, were originally defined over a field, but the same definitions still apply when the matrix entries are from any ring $R$. The ring of all $\omega \times \omega$ matrices over $R$ which are simultaneously row-finite and column-finite is denoted by $B(R)$. For a matrix $x \in B(R)$, a growth curve for $x$ is any function $g: \mathbb{N} \rightarrow \mathbb{R}^{+}$such that for each $n \in \mathbb{N}, x(n, i)=0=x(i, n)$ for all $i>n+g(n)$. (So $g(n)$ gives a bound on the "bandwidth" of $x$ at the $(n, n)$ position.) The matrix $x$ has $O(g(n))$ growth if there is a constant $c>0$ such that the function $c g(n)$ is a growth curve for $x$. Now for any $r \in[0,1]$, the ring $G_{R}(r)$ can be defined as

$$
G_{R}(r)=\left\{x \in B(R): x \text { has } O\left(n^{r}\right) \text { growth }\right\} .
$$

It will be shown in Section 3 that these rings are translation rings over $R$ relative to a suitable pseudo-metric on $\mathbb{N}$. In the case where $R=F$ is a field and $A$ is a countable-dimensional algebra over $F$, the bandwidth dimension of $A$ is defined as

$$
\inf \left\{r \in \mathbb{R}^{+}: A \text { embeds in } G_{F}(r)\right\} .
$$

It was shown in [13] that the bandwidth dimensions of even finitely generated algebras exactly fill the unit interval $[0,1]$.

Following Warfield [18, we call a ring $R$ an exchange ring if $R_{R}$ satisfies the finite exchange property. In fact, this is equivalent to saying that for any finitely generated projective module $M_{R}$ and internal $R$-module decompositions $A=M^{\prime} \oplus$ $N=\bigoplus_{i=1}^{n} A_{i}$ with $M^{\prime} \cong M$, there exist submodules $A_{i}^{\prime}$ of $A_{i}$ such that $A=$ $M^{\prime} \oplus\left(\bigoplus_{i=1}^{n} A_{i}^{\prime}\right)$. The class of exchange rings is quite large, including all semiregular rings (rings which are regular modulo their Jacobson radical and have idempotentlifting), all $\pi$-regular rings, all $C^{*}$-algebras with real rank zero, and more. See 1, 3, 17, 18. The following element-wise criterion for exchange rings was obtained independently by Goodearl [8, p. 167] and Nicholson [12, Theorem 2.1], and is particularly useful.

Proposition 1.1 (Goodearl and Nicholson). A ring $R$ is an exchange ring if and only if for every element $a \in R$ there exists an idempotent $f \in R$ such that $f \in a R$ and $1-f \in(1-a) R$.

We recall that a ring $R$ is (von Neumann) regular if for each $a \in R$ there exists $b \in R$ with $a=a b a$. Equivalently, every one-sided principal ideal of $R$ can be generated by a single idempotent. Reference [6] remains the authoritative source for both the theory and examples of regular rings. It is an old and easy fact that regular rings are exchange rings (see [18, Theorem 3]; this also follows from Proposition 1.2 below by taking $M=R, A=a R$ and $B=(1-a) R$ for $a \in R)$. However, the exchange rings arising as a translation ring $T(X, R)$ of an (infinite) discrete tree $X$ over a regular ring $R$ are not themselves regular rings. 
We complete our preliminaries by recalling the following well-known technique for producing a direct sum decomposition over a regular ring.

Proposition 1.2. Let $M$ be a projective module over a regular ring $R$. Suppose $A$ and $B$ are submodules of $M$ such that $A \cap B$ is finitely generated. (Note that this certainly holds if both $A$ and $B$ are finitely generated.) Then there exists a direct summand $C$ of $A$ such that

$$
A+B=C \oplus B .
$$

Proof. Since $A \cap B$ is finitely generated, it is a direct summand of the projective module $M$ by [6] Theorem 1.11]. Write $A=(A \cap B) \oplus C$. Then $A+B=C \oplus B$.

\section{The MAIN RESUlT}

Throughout this section $R$ denotes a (von Neumann) regular ring, $(X, d)$ a locally finite metric space, and $T=T(X, R)$ the translation ring of $X$ over $R$. Although $T$ is defined in terms of certain infinite matrices, a transformation viewpoint suits our proofs better. Accordingly, we fix a free right $R$-module $V$ and a basis $\left\{e_{x}\right\}_{x \in X}$ for $V$, and we identify $T$ with the ring of all $R$-endomorphisms of $V_{R}$ whose matrix relative to the basis $\left\{e_{x}\right\}_{x \in X}$ has constant bandwidth $\ell$ for some $\ell \geq 0$ (so for $a \in T$ we have $a\left(e_{x}\right)=\sum_{y \in X} e_{y} a(y, x)$, where $a(y, x)=0$ whenever $\left.d(x, y)>\ell\right)$. We also fix the following notation.

Notation. For any subset $Y$ of $X$, let $V(Y)$ be the $R$-submodule of $V$ generated by the $e_{y}$ with $y \in Y$. For $x \in X$ and $r \in \mathbb{Z}^{+}$, let

$$
\begin{aligned}
& B(x, r)=\{y \in X: d(x, y)<r\} \quad \text { (the open ball), } \\
& V(x, r)=V(B(x, r)) .
\end{aligned}
$$

In preparation for the proof of our main result in Theorem 2.7, we assemble six lemmas. The first formulates bandwidth in transformation terms and will be used frequently.

Lemma 2.1. Suppose $a \in T$ has bandwidth $\ell$. Then:

(1) For any basis element $e_{x}$, the support of $a\left(e_{x}\right)$ does not contain basis elements $e_{y}$ with $d(y, x)>\ell$.

(2) If $k>0$ and $c \in V(x, k)$, then $a(c) \in V(x, k+\ell)$. (The closed ball version of this is also true.)

Proof. (1) $a\left(e_{x}\right)=\sum_{y} e_{y} a(y, x)$, where the matrix $(a(y, x))$ has bandwidth $\ell$. Hence $a(y, x)=0$ when $d(y, x)>\ell$.

(2) It is enough to establish this for $c=e_{y}$ with $d(x, y)<k$. But it follows from (1) that $d(y, z) \leq \ell$ for any $e_{z}$ in the support of $a\left(e_{y}\right)$, and so $d(x, z) \leq$ $d(x, y)+d(y, z)<k+\ell$.

The next lemma gives a criterion for containment of principal right ideals of a translation ring.

Lemma 2.2. Let $\left\{B\left(x_{i}, k\right)\right\}_{i=1}^{\infty}$ be a covering of $X$ by balls of radius $k>0$. For $a, b \in T$, the containment $a T \subseteq b T$ holds if and only if there exists $m \in \mathbb{N}$ such that $a V\left(x_{i}, k\right) \subseteq b V\left(x_{i}, m\right)$ for all $i$. 
Proof. $(\Rightarrow)$ Assume $a=b c$ with $c \in T$ of bandwidth $\ell$. By Lemma 2.1(2), $a V\left(x_{i}, k\right)=b\left(c V\left(x_{i}, k\right)\right) \subseteq b V\left(x_{i}, k+\ell\right)$.

$(\Leftarrow)$ Write $X=\bigcup_{i=1}^{\infty} B_{i}$ as a disjoint union with $B_{i} \subseteq B\left(x_{i}, k\right)$. Then $V=$ $\bigoplus_{i=1}^{\infty} V_{i}$, where $V_{i}=V\left(B_{i}\right)$. Since $a V_{i} \subseteq b V\left(x_{i}, m\right)$, there exists an $R$-submodule $W_{i}$ of $V\left(x_{i}, m\right)$ such that $a V_{i}=b W_{i}$. Set $a_{i}=\left.a\right|_{V_{i}}$ and $b_{i}=\left.b\right|_{W_{i}}$. By projectivity of $V_{i}$, there is an $R$-module map $c_{i}: V_{i} \rightarrow W_{i}$ such that the diagram

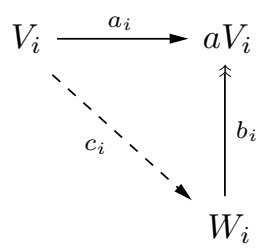

commutes for all $i$. Set $c=\bigoplus_{i} c_{i}$. Obviously $a=b c$, but we need to verify that $c \in T$. Fix $x \in X$, say $x \in B_{i}$. By construction $c\left(e_{x}\right) \in W_{i} \subseteq V\left(x_{i}, m\right)$, so $c\left(e_{x}\right)=\sum_{d\left(y, x_{i}\right)<m} e_{y} c(y, x)$. But $d\left(x, x_{i}\right)<k$, whence for the $e_{y}$ in the support of $c\left(e_{x}\right)$, we have $d(y, x) \leq d\left(y, x_{i}\right)+d\left(x_{i}, x\right)<m+k$. Thus $c \in T$ with bandwidth less than $m+k$.

For the remainder of this section, we specialize our locally finite metric space to the case where $X$ is a discrete tree and the metric $d$ is the path metric:

$$
d(x, y)=\text { length of the (unique) path joining } x \text { and } y .
$$

We also fix the following notation.

\section{Notation.}

$a=$ fixed element of $T=T(X, R)$.

$\ell=$ bandwidth of $a$.

$x_{0}=$ fixed element of $X$.

$\gamma(x)=$ (set of vertices along) the geodesic from $x_{0}$ to $x$ (for any $x \in X$ ).

$U_{x}(k)=V\left(\left\{y \in X: x \in \gamma(y)\right.\right.$ and $\left.\left.d\left(y, x_{0}\right) \geq d\left(x, x_{0}\right)+k\right\}\right)$ for any $x \in X$ and $k \in \mathbb{Z}^{+}$.

$W_{x}(k)=V\left(\left\{y \in X: x \in \gamma(y)\right.\right.$ and $\left.\left.k \ell \leq d\left(y, x_{0}\right)<(k+1) \ell\right\}\right)$ for any $x \in X$ and $k \in \mathbb{Z}^{+}$.

$S_{x}=a U_{x}(\ell)+(1-a) U_{x}(\ell)$ for any $x \in X$.

Lemma 2.3. For any $k \in \mathbb{Z}^{+}$,

$$
\begin{aligned}
& V\left(x_{0},(k+2) \ell\right) \cap\left(\sum_{d\left(x_{0}, y\right)=k \ell} S_{y}\right) \\
& \quad \subseteq \sum_{d\left(x_{0}, y\right)=k \ell}\left[a\left(W_{y}(k+1)+W_{y}(k+2)\right)+(1-a)\left(W_{y}(k+1)+W_{y}(k+2)\right)\right] .
\end{aligned}
$$

Proof. Suppose $v=\sum_{d\left(x, x_{0}\right)<(k+2) \ell} e_{x} \alpha_{x} \in \sum_{d\left(x_{0}, y\right)=k \ell} S_{y}$. Observe that

$$
S_{y}=a W_{y}(k+1)+(1-a) W_{y}(k+1)+\sum_{j \geq 2}\left[a W_{y}(k+j)+(1-a) W_{y}(k+j)\right]
$$


for each $y$ with $d\left(x_{0}, y\right)=k \ell$. Notice that the square bracketed terms make no contribution to $V\left(x_{0},(k+1) \ell\right)$ by Lemma 2.1. Hence,

$$
v=a w+(1-a) w^{\prime}+v^{\prime}
$$

where

$$
w, w^{\prime} \in \sum_{d\left(x_{0}, y\right)=k \ell} W_{y}(k+1)
$$

and

$$
v^{\prime} \in \sum_{(k+1) \ell \leq d\left(x, x_{0}\right)<(k+3) \ell} e_{x} R \subseteq \sum_{d\left(x_{0}, y\right)=k \ell}\left[W_{y}(k+1)+W_{y}(k+2)\right] .
$$

Since $W_{y}(m) \subseteq a W_{y}(m)+(1-a) W_{y}(m)$ for any $m$, it follows that

$$
v \in \sum_{d\left(x_{0}, y\right)=k \ell}\left[a\left(W_{y}(k+1)+W_{y}(k+2)\right)+(1-a)\left(W_{y}(k+1)+W_{y}(k+2)\right)\right]
$$

as desired.

The four properties of the submodules $S_{x}$ recorded in the next lemma make critical use of the fact that $X$ is a tree.

Lemma 2.4. Let $k \in \mathbb{Z}^{+}$.

(1) Let $x \in X$. For any $e_{z}$ in the support of $S_{x}$, we have $x \in \gamma(z)$.

(2) The sum $\sum_{d\left(x, x_{0}\right)=k} S_{x}$ is a direct sum.

(3) Let $x \in X$. Then

$$
\bigoplus_{\substack{x \in \gamma(y), d\left(y, x_{0}\right)=d\left(x, x_{0}\right)+k}} S_{y} \subseteq U_{x}(k) .
$$

(4) Let $y \in X, t \geq d\left(y, x_{0}\right)$. Then

$$
S_{y}=a W+(1-a) W+\left(\bigoplus_{\substack{y \in \gamma(z), d\left(z, x_{0}\right)=t}} S_{z}\right),
$$

where

$$
W=V\left(\left\{w \in X \mid y \in \gamma(w), d\left(y, x_{0}\right)+\ell \leq d\left(w, x_{0}\right)<t+\ell\right\}\right) .
$$

Proof. (1) $S_{x}$ is generated by elements of the form $a\left(e_{y}\right)$ or $(1-a)\left(e_{y}\right)$, where $y \in X$ is of distance at least $\ell$ further from $x_{0}$ than $x$, and $x$ lies on the geodesic from $x_{0}$ to $y$. By Lemma 2.1, if $e_{z}$ is in the support of $a\left(e_{y}\right)$ or $(1-a)\left(e_{y}\right)$, then $d(z, y) \leq \ell$. Since $X$ is a tree, this places $x$ on $\gamma(z)$ too, as follows.

Assume, by way of contradiction, that $x \notin \gamma(z)$. Let $\gamma^{\prime}$ be the geodesic joining $z$ and $y$. Then $\gamma(y)$ is the reduced path obtained from the composition of $\gamma(z)$ and $\gamma^{\prime}$. Since $d(y, z) \leq \ell$, every vertex in $\gamma^{\prime}$ is different from $x$. Therefore $x \notin \gamma(y)$, which is impossible.

Therefore, for any two different $x$ with the same $d\left(x_{0}, x\right)$, their $S_{x}$ must have disjoint supports. Thus (2) holds. (3) is similar, and (4) is straightforward.

So far in this section we have made no special use of the fact that the ring $R$ is regular. However, regularity is used a little in the following lemma, and will be put to full use in Lemma 2.6. 
Lemma 2.5. Let $x \in X$ and $r \in \mathbb{Z}^{+}$with $r \geq d\left(x, x_{0}\right)$. Let

$$
N=\bigoplus_{\substack{x \in \gamma(y) \\ d\left(y, x_{0}\right)=r}} S_{y} .
$$

Suppose $B$ is a submodule of $V_{R}$ such that $B \supseteq N$ and $B / N$ is finitely generated. Then for any finitely generated submodule $A$ of $V_{R}, A \cap B$ is also finitely generated.

Proof. We can write $B=G+N$ for some finitely generated submodule $G$ of $V$. Let $s=d\left(x, x_{0}\right)$. Choose $k \geq r+\ell-s$ such that $A, G \subseteq V\left(x_{0}, k\right)$. We claim that $B \subseteq V\left(x_{0}, k+s\right) \oplus U_{x}(k)$.

In order to see this it is enough to check that $S_{y} \subseteq V\left(x_{0}, k+s\right) \oplus U_{x}(k)$ whenever $x \in \gamma(y)$ and $d\left(y, x_{0}\right)=r$. By condition (1) in Lemma 2.4, if $e_{z}$ is in the support of $S_{y}$, then $y \in \gamma(z)$, hence also $x \in \gamma(z)$. If $d\left(x_{0}, z\right)<k+s$, then $e_{z} \in V\left(x_{0}, k+s\right)$, and $e_{z} \in U_{x}(k)$ otherwise.

We also have that $U_{x}(k) \subseteq N \subseteq B$ (because $x \in \gamma(u)$ and $d\left(u, x_{0}\right) \geq d\left(x, x_{0}\right)+k$ imply $d\left(u, x_{0}\right) \geq r+\ell$, and therefore $e_{u} \in U_{y}(\ell)$ for some $y$ with $x \in \gamma(y), d\left(y, x_{0}\right)=$ $r$, so $\left.e_{u} \in S_{y} \subseteq N\right)$. Let $Z=B \cap V\left(x_{0}, k+s\right)$. By the modular law, $B=Z \oplus U_{x}(k)$ and so $Z \cong B / U_{x}(k)$.

Now let

$$
L=\bigoplus_{\substack{x \in \gamma(z) \\ d\left(z, x_{0}\right)=k+s}} S_{z} .
$$

By Lemma 2.4(3), we have $U_{x}(k) \supseteq L$. By Lemma 2.4(4) with $t=k+s$, we have $N=a W+(1-a) W+L$, where

$$
W=V\left(\left\{w \in X: x \in \gamma(w) \text { and } r+\ell \leq d\left(w, x_{0}\right)<k+s+\ell\right\}\right) .
$$

Therefore we get

$$
B=G+N=G+a W+(1-a) W+L .
$$

It follows that $B / L$ is finitely generated and, since $L \subseteq U_{x}(k)$, so is $B / U_{x}(k) \cong Z$. Now $A \cap B=A \cap Z$ is the intersection of two finitely generated submodules of the projective module $V$, and so $A \cap B$ is finitely generated because $R$ is regular [6] Lemma 2.2].

Lemma 2.6. For each $x \in X$ with $d\left(x, x_{0}\right)=4 n \ell$ for some $n \in \mathbb{Z}^{+}$, there exist $R$-submodules $C_{x}, D_{x}$ of $V$ such that:

(1) $S_{x}=C_{x} \oplus D_{x} \oplus\left(\underset{\substack{x \in \gamma(y) \\ d\left(y, x_{0}\right)=4(n+1) \ell}}{\bigoplus} S_{y}\right)$.

(2) $C_{x} \subseteq a\left[W_{x}(4 n+1)+W_{x}(4 n+2)+W_{x}(4 n+3)+W_{x}(4 n+4)\right]$, $D_{x} \subseteq(1-a)\left[W_{x}(4 n+1)+W_{x}(4 n+2)+W_{x}(4 n+3)+W_{x}(4 n+4)\right]$.

(3) $W_{x}(4 n+1)+W_{x}(4 n+2)+W_{x}(4 n+3)+W_{x}(4 n+4)$

$$
\subseteq C_{x} \oplus D_{x} \oplus\left(\underset{\substack{x \in \gamma(y) \\ d\left(y, x_{0}\right)=4(n+1) \ell}}{\bigoplus} C_{y} \oplus D_{y}\right) .
$$

Proof. Let $x \in X$ with $d\left(x, x_{0}\right)=4 n \ell$ for some $n \in \mathbb{Z}^{+}$. Since

$$
a\left(W_{x}(4 n+1)+W_{x}(4 n+2)\right) \text { and }(1-a)\left(W_{x}(4 n+1)+W_{x}(4 n+2)\right)
$$


are finitely generated submodules of $V_{R}$, by Proposition 1.2 we can write

$$
a\left(W_{x}(4 n+1)+W_{x}(4 n+2)\right)+(1-a)\left(W_{x}(4 n+1)+W_{x}(4 n+2)\right)=C_{x}^{\prime} \oplus D_{x}^{\prime}
$$

for some finitely generated submodules $C_{x}^{\prime} \subseteq a\left(W_{x}(4 n+1)+W_{x}(4 n+2)\right)$ and $D_{x}^{\prime} \subseteq(1-a)\left(W_{x}(4 n+1)+W_{x}(4 n+2)\right)$. Let

$$
\begin{aligned}
N= & \bigoplus_{\substack{x \in \gamma(y) \\
d\left(y, x_{0}\right)=(4 n+4) \ell}} S_{y}, \\
A= & a\left(W_{x}(4 n+3)+W_{x}(4 n+4)\right), \\
B= & a\left(W_{x}(4 n+1)+W_{x}(4 n+2)\right)+(1-a)\left(W_{x}(4 n+1)+W_{x}(4 n+2)\right)+N .
\end{aligned}
$$

Notice that $B=C_{x}^{\prime} \oplus D_{x}^{\prime} \oplus N$, because by Lemma 2.1 $C_{x}^{\prime}, D_{x}^{\prime} \subseteq V\left(x_{0},(4 n+4) \ell\right)$ and $V\left(x_{0},(4 n+4) \ell\right) \cap N=0$ (by Lemma 2.1(1)). Also because $A$ and $B / N$ are finitely generated, $A \cap B$ is finitely generated by Lemma 2.5 whence by Proposition 1.2 there exists a finitely generated submodule $C_{x}^{\prime \prime}$ of $A$ such that $A+B=$ $C_{x}^{\prime \prime} \oplus B$. Let

$$
\begin{aligned}
& A^{\prime}=(1-a)\left(W_{x}(4 n+3)+W_{x}(4 n+4)\right), \\
& B^{\prime}=A+B=C_{x}^{\prime \prime} \oplus B .
\end{aligned}
$$

Again by Lemma2.5, we have that $A^{\prime} \cap B^{\prime}$ is finitely generated because $A^{\prime}$ and $B^{\prime} / N$ are finitely generated. Therefore, by Proposition 1.2 we can write $A^{\prime}+B^{\prime}=D_{x}^{\prime \prime} \oplus B^{\prime}$ for some summand $D_{x}^{\prime \prime}$ of $A^{\prime}$.

By Lemma 2.4 4$)$ with $t=(4 n+4) \ell$, we have

$$
S_{x}=a\left(\sum_{k=4 n+1}^{4 n+4} W_{x}(k)\right)+(1-a)\left(\sum_{k=4 n+1}^{4 n+4} W_{x}(k)\right)+N .
$$

By using the above calculations we get

$$
\begin{aligned}
S_{x}= & a\left(\sum_{k=4 n+1}^{4 n+4} W_{x}(k)\right)+(1-a)\left(\sum_{k=4 n+1}^{4 n+4} W_{x}(k)\right)+N \\
= & a\left(W_{x}(4 n+1)+W_{x}(4 n+2)\right)+(1-a)\left(W_{x}(4 n+1)+W_{x}(4 n+2)\right) \\
& +N+A+A^{\prime} \\
= & B+A+A^{\prime} \\
= & \left(\left(C_{x}^{\prime} \oplus D_{x}^{\prime} \oplus N\right)+A\right)+A^{\prime} \\
= & \left(C_{x}^{\prime} \oplus D_{x}^{\prime} \oplus N \oplus C_{x}^{\prime \prime}\right)+A^{\prime} \\
= & C_{x}^{\prime} \oplus D_{x}^{\prime} \oplus N \oplus C_{x}^{\prime \prime} \oplus D_{x}^{\prime \prime} \\
= & \left(C_{x}^{\prime} \oplus C_{x}^{\prime \prime}\right) \oplus\left(D_{x}^{\prime} \oplus D_{x}^{\prime \prime}\right) \oplus N .
\end{aligned}
$$

Thus, setting $C_{x}=C_{x}^{\prime} \oplus C_{x}^{\prime \prime}$ and $D_{x}=D_{x}^{\prime} \oplus D_{x}^{\prime \prime}$ achieves not only goals (1) and (2) (for all $x$ whose distance from $x_{0}$ is a multiple of $4 \ell$ ) but a bit more - the following holds:

(*) $a\left(W_{x}(4 n+1)+W_{x}(4 n+2)\right)+(1-a)\left(W_{x}(4 n+1)+W_{x}(4 n+2)\right) \subseteq C_{x} \oplus D_{x}$. 
We now show that (3) follows. Again fix $x \in X$ with $d\left(x, x_{0}\right)=4 n \ell$. Let

$$
Q=\left\{y \in X: d\left(y, x_{0}\right)=4(n+1) \ell \text { and } x \in \gamma(y)\right\},
$$

and let

$$
\pi: S_{x}=C_{x} \oplus D_{x} \oplus N \rightarrow N
$$

be the projection onto $N=\bigoplus_{y \in Q} S_{y}$. Observe that $W_{x}(4 n+1)+\cdots+W_{x}(4 n+4)$ is a submodule of $U_{x}(\ell)$ (hence of $\left.S_{x}\right)$ and $V\left(x_{0},(4 n+5) \ell\right.$ ). Also, by (2) and Lemma 2.1, $C_{x}$ and $D_{x}$ are submodules of $V\left(x_{0},(4 n+6) \ell\right)$. Therefore by Lemma 2.3 ,

$$
\begin{aligned}
& \pi\left(W_{x}(4 n+1)+\cdots+W_{x}(4 n+4)\right) \subseteq V\left(x_{0},(4 n+6) \ell\right) \cap \sum_{y \in Q} S_{y} \\
& \quad \subseteq \sum_{y \in Q}\left[a\left(W_{y}(4 n+5)+W_{y}(4 n+6)\right)+(1-a)\left(W_{y}(4 n+5)+W_{y}(4 n+6)\right)\right] \\
& \subseteq \bigoplus_{y \in Q}\left(C_{y} \oplus D_{y}\right) \quad \text { by property }(*) .
\end{aligned}
$$

Property (3) is now evidently true.

We are now ready to state and prove our main result.

Theorem 2.7. Let $X$ be a discrete tree and $R$ any regular ring. Then the translation ring $T(X, R)$ is an exchange ring.

Proof. Let $T=T(X, R)$. To show $T$ is an exchange ring, it suffices by Proposition 1.1 to demonstrate that for each $a \in T$, there is an idempotent $f \in T$ with $f \in a T$ and $1-f \in(1-a) T$. So fix $a \in T$ with bandwidth $\ell$, and perform the construction in Lemma 2.6 to produce $C_{x}, D_{x}$ for each $x \in X$ with $d\left(x, x_{0}\right)=4 n \ell$, $n \in \mathbb{Z}^{+}$.

Let $W_{0}=V\left(x_{0}, \ell\right)$ and $N=S_{x_{0}}$. By observing that $V=a W_{0}+(1-a) W_{0}+$ $N$, and utilizing Lemma 2.5 (as in the proof of Lemma 2.6), we can find finitely generated $R$-submodules $C_{0} \subseteq a W_{0}$ and $D_{0} \subseteq(1-a) W_{0}$ such that $V=C_{0} \oplus D_{0} \oplus N$. Setting $P=\left\{x \in X: d\left(x, x_{0}\right)=4 n \ell\right.$ for some $\left.n \in \mathbb{Z}^{+}\right\}$, we claim that

$$
V=C_{0} \oplus D_{0} \oplus\left[\bigoplus_{x \in P}\left(C_{x} \oplus D_{x}\right)\right] .
$$

Indeed, the sum is direct by (1) of Lemma 2.6 (and 2.4(2)). To see that equality holds, note first that Lemma 2.6(3) implies $U_{x_{0}}(4 \ell) \subseteq E:=\bigoplus_{x \in P}\left(C_{x} \oplus D_{x}\right)$. Now we have

$$
\begin{array}{rlrl}
V & =C_{0} \oplus D_{0} \oplus N=C_{0} \oplus D_{0} \oplus S_{x_{0}} & \\
& =C_{0} \oplus D_{0} \oplus C_{x_{0}} \oplus D_{x_{0}} \oplus \bigoplus_{d\left(y, x_{0}\right)=4 \ell} S_{y} \quad \text { (by Lemma[2.6(1)) } \\
& \subseteq\left(C_{0} \oplus D_{0} \oplus C_{x_{0}} \oplus D_{x_{0}}\right)+U_{x_{0}}(4 \ell) & \text { (by Lemma[2.4(3)) } \\
& \subseteq C_{0} \oplus D_{0} \oplus E,
\end{array}
$$

which establishes our claim. Let $C=C_{0} \oplus\left(\bigoplus_{x \in P} C_{x}\right), D=D_{0} \oplus\left(\bigoplus_{x \in P} D_{x}\right)$, and let

$$
f: V=C \oplus D \rightarrow C
$$

be the projection. 
Claim. There exists $m \in \mathbb{N}$ such that for all $z \in X$,

$$
f\left(e_{z}\right) \in a V(z, m) \quad \text { and } \quad(1-f)\left(e_{z}\right) \in(1-a) V(z, m) .
$$

To verify this, fix $z \in X$. If $e_{z} \in W_{0}$, there is no problem arranging a bound $m$, because surely $f\left(e_{z}\right) \in a V$ and $(1-f)\left(e_{z}\right) \in(1-a) V$, so there exists $M \in \mathbb{N}$ with $f\left(e_{z}\right) \in a V\left(x_{0}, M\right)$ and $(1-f)\left(e_{z}\right) \in(1-a) V\left(x_{0}, M\right)$ for all (the finitely many) $e_{z} \in W_{0}$. Hence $m=M+\ell$ will do. (The argument at the end of Lemma 2.6 produces a better bound, $m=6 \ell$.) So we can assume $e_{z} \in W_{x}(4 n+j)$ for some $x \in X$ with $d\left(x, x_{0}\right)=4 n \ell, n \in \mathbb{Z}^{+}$, and some $j=1,2,3,4$. Let

$$
Q=\left\{y \in X: x \in \gamma(y) \text { and } d\left(y, x_{0}\right)=4(n+1) \ell\right\} .
$$

By (3) of Lemma 2.6,

$$
W_{x}(4 n+j) \subseteq C_{x} \oplus D_{x} \oplus\left[\bigoplus_{y \in Q}\left(C_{y} \oplus D_{y}\right)\right],
$$

whence

$$
\begin{aligned}
f\left(W_{x}(4 n+j)\right) \subseteq & C_{x} \oplus\left(\bigoplus_{y \in Q} C_{y}\right) \\
\subseteq & a\left[\left(W_{x}(4 n+1)+\cdots+W_{x}(4 n+4)\right)\right. \\
& \left.+\sum_{y \in Q}\left(W_{y}(4 n+5)+\cdots+W_{y}(4 n+8)\right)\right] .
\end{aligned}
$$

It is at this point that we make yet another key use of the fact that $X$ is a tree, not just a connected graph. Namely, when $x \in \gamma(y)$, then $d(x, y)=d\left(y, x_{0}\right)-$ $d\left(x, x_{0}\right)$. Therefore, for all $e_{z^{\prime}}$ in the support of the last square bracketed term, $d\left(x, z^{\prime}\right)<9 \ell$. On the other hand, $d(x, z)<5 \ell$, whence $d\left(z, z^{\prime}\right)<14 \ell$. This shows that $f\left(e_{z}\right) \in a V(z, 14 \ell)$. Analogously, $(1-f)\left(e_{z}\right) \in(1-a) V(z, 14 \ell)$, so taking $m=\max \{M+\ell, 14 \ell\}$ satisfies the claim.

The claim yields two things. Firstly, $f \in T$ because $f\left(e_{z}\right) \in a V(z, m)$ implies $f\left(e_{z}\right) \in V(z, \ell+m)$ for all $z \in X$ by Lemma 2.1, and so $f$ has constant bandwidth at most $\ell+m$. Secondly, $f \in a T$ and $1-f \in(1-a) T$ by Lemma 2.2 (taking $k=1$ ).

This completes our proof that $T$ is an exchange ring.

Remark 2.8. As regards weakening the regularity hypothesis on $R$ in Theorem 2.7 one certainly needs $R$ to be at least an exchange ring in order for $T(X, R)$ to be such, because $R$ is a corner ring ( $e T e$ for some idempotent $e \in T$ ) of the latter. On the other hand, $R$ being an exchange ring is not enough on its own. For example, one can show that if the ring $G_{R}(0)$ (of $\omega \times \omega$ matrices over $R$ of constant bandwidth) is an exchange ring, then the Jacobson radical of $R$ must be right and left $T$-nilpotent.

Our theorem naturally prompts the following question:

Question. Does Theorem 2.7 hold if $X$ is a connected graph? What about a locally finite metric space? A good test case might be when $X$ is the Cayley graph of a free abelian group, even $\mathbb{Z} \oplus \mathbb{Z}$. (Note that 2.7 does apply to the Cayley graph of a free non-abelian group.) 


\section{The Growth algebras $G(r)$}

In this section we apply our results of Section 2 to show that the rings $G_{R}(r)$ are exchange rings for all regular rings $R$ and for all $r \in[0,1]$. Theorem [2.7] as it stands, only applies directly to $G_{R}(0)$. However, with very little extra work, it can be extended in the following way, which will then apply directly to all the $G_{R}(r)$.

Theorem 3.1. Let $X$ be a set and let $\sim$ be an equivalence relation on $X$ such that all the equivalence classes are finite and the quotient set $X / \sim$ has a discrete tree structure. Let $d$ be the induced pseudo-metric on $X$, that is,

$$
d(x, y)=\text { length of the path from }[x] \text { to }[y]
$$

(where [ ] denotes the $\sim$ equivalence class). Then the translation ring $T(X, R)$ is an exchange ring for all regular rings $R$.

Proof. Notice that Lemmas 2.1 and 2.2 work for any locally finite pseudo-metric space. In the remainder of Section 2, the submodules $U_{x}(k), W_{x}(k), S_{x}, C_{x}$ and $D_{x}$ are now to be indexed by the equivalence classes $[x]$, but their definitions (which are independent of the representative of $[x]$ ) target the elements of $X$, not $X / \sim$. Thus,

$$
\begin{aligned}
U_{[x]}(k) & =V\left(\left\{y \in X:[x] \in \gamma([y]) \text { and } d\left(y, x_{0}\right) \geq d\left(x, x_{0}\right)+k\right\}\right), \\
W_{[x]}(k) & =V\left(\left\{y \in X:[x] \in \gamma([y]) \text { and } k \ell \leq d\left(y, x_{0}\right)<(k+1) \ell\right\}\right), \\
S_{[x]} & =a U_{[x]}(\ell)+(1-a) U_{[x]}(\ell) .
\end{aligned}
$$

One then easily checks that, with some very minor changes, the statements and proofs of 2.3 2.4 2.5, 2.6 and 2.7 carry over intact.

Given any sequence $S=\left\{n_{i}\right\}_{1}^{\infty}$ of (strictly) positive integers, we can define a pseudo-metric $d_{S}$ on $\mathbb{N}$ by taking $d_{S}(x, y)=|i-j|$, where $n_{1}+\cdots+n_{i-1}<x \leq$ $n_{1}+\cdots+n_{i}$ and $n_{1}+\cdots+n_{j-1}<y \leq n_{1}+\cdots+n_{j}$ (set $n_{0}=0$ ). Modulo the obvious equivalence on $\mathbb{N}$ (that induced by the intervals of integers $\left(0, n_{1}\right],\left(n_{1}, n_{1}+n_{2}\right]$, $\left.\left(n_{1}+n_{2}, n_{1}+n_{2}+n_{3}\right], \ldots\right), d_{S}$ is just the Euclidean metric. (The distance between $x$ and $y$ is the number of "blocks" $\left(n_{1}+\cdots+n_{i-1}, n_{1}+\cdots+n_{i}\right]$ they are apart.) Hence Theorem 3.1 applies, and so the corresponding translation $\operatorname{ring} T_{S}(R)=T(\mathbb{N}, R)$ is an exchange ring for any regular ring $R$. There is a simple description of the matrices in $T_{S}(R)$. Consider the natural copy of $\prod_{i=1}^{\infty} M_{n_{i}}(R)$ inside $B(R)$, that is, all blockdiagonal matrices with block sizes $n_{1}, n_{2}, n_{3}, \ldots$ (going down the diagonal). (In [11] this was called the "spine" determined by the sequence $\left\{n_{i}\right\}$.) Then $T_{S}(R)$ consists of all $\omega \times \omega$ matrices over $R$ which have constant "block-bandwidth", that is, when bandwidth is measured in terms of the number of off-diagonal blocks, rather than the number of off-diagonal entries. See [11, p. 116]. For example, if $n_{1}=1, n_{2}=2$, $n_{3}=2, n_{4}=3, \ldots$ the matrix below has block-bandwidth 1 :

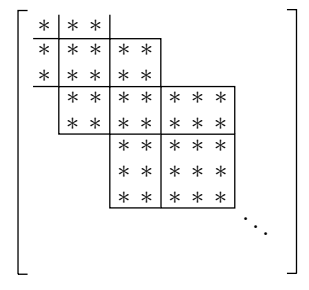


Each of the rings $G_{R}(r)$ (over any coefficient ring $R$ ) is of the form $T_{S}(R)$ for some suitable sequence $S=\left\{n_{i}\right\}_{1}^{\infty}$ : for $r=1$, we can take $n_{i}=2^{i}$; for $0 \leq r<1$, let $t=r /(1-r)$ and take $n_{i}=\left[i^{t}\right]$, the greatest integer less than or equal to $i^{t}$. See [11, Proposition 1.6]. Therefore, Theorem 3.1yields the following corollary.

Corollary 3.2. The rings $G_{R}(r)$ are exchange rings for all regular rings $R$ and for all $r \in[0,1]$.

It is not difficult to see that the $\operatorname{ring} B(R)$, of all $\omega \times \omega$ row-and-column-finite matrices over a ring $R$, can never be a translation ring relative to any locally finite pseudo-metric $d$ on $\mathbb{N}$. However, each matrix in $B(R)$ is block tridiagonal relative to suitably sized blocks (depending on the matrix), and therefore

$$
B(R)=\bigcup_{S} T_{S}(R),
$$

where the union is over all sequences $S=\left\{n_{i}\right\}$ of positive integers. Thus Theorem 3.1 yields as a further corollary the following result, first established in [14], because clearly a ring which is a union of exchange rings is an exchange ring by Proposition 1.1.

Corollary 3.3. The ring $B(R)$ is an exchange ring for all regular rings $R$.

Our final corollary helps quantify the wide range of exchange algebras produced by our constructions.

Corollary 3.4. (1) Over any field $F$, and for any real number $r \in[0,1]$, there is an exchange algebra of bandwidth dimension $r$.

(2) For a countable field $F$, the exchange algebra in (1) can be chosen to be countable-dimensional.

Proof. (1) For this statement, we can take the algebra $G_{F}(r)$ and appeal to Corollary 3.2 and [13, Corollary 8.1].

(2) Now suppose $F$ is a countable field and $r \in[0,1]$. By [13. Theorem 0.1] there is a finitely generated $F$-algebra $A \subseteq G_{F}(r)$ of bandwidth dimension $r$. Of course, since $F$ is countable, $A$ (and any countable-dimensional algebra) is countable. Also, by Corollary 3.2 , we know $G_{F}(r)$ is an exchange algebra. There is now a standard way of extending $A$ to a countable subalgebra $B$ of $G_{F}(r)$ such that $B$ is an exchange algebra, using the condition in Proposition 1.1. Namely, we take $B$ as the union of a chain $A_{1}=A \subseteq A_{2} \subseteq$. of subalgebras of $G_{F}(r)$ constructed inductively as follows: If $A_{n}=\left\{a_{1}, a_{2}, a_{3}, \ldots\right\}$, choose idempotents $f_{1}, f_{2}, f_{3}, \ldots$ in $G_{F}(r)$ such that $f_{i}=a_{i} x_{i}$ and $1-f_{i}=\left(1-a_{i}\right) y_{i}$ for some $x_{i}, y_{i} \in G_{F}(r)$, and let $A_{n+1}$ be the $F$-subalgebra generated by $A_{n}$ and $f_{i}, x_{i}, y_{i}$ for $i=1,2,3, \ldots$.

That $B$ has bandwidth dimension $r$ follows from the squeezing $A \subseteq B \subseteq G_{F}(r)$, because both $A$ and $G_{F}(r)$ are of that dimension.

Remark 3.5. In contrast to Corollary 3.4 it appears to be an open question whether there are countable dimensional (von Neumann) regular algebras of arbitrary bandwidth dimension $r \in[0,1]$.

\section{REFERENCES}

[1] P. ArA, Extensions of exchange rings, J. Algebra 197 (1997), 409-423. MR 98j:16021

[2] P. ArA, Stability properties of exchange rings, in: "International Symposium on Ring Theory”, (Kyongju, 1999), Trends Math., Birkhäuser, Boston, 2001, pp. 23-42. MR 2002g:16012 
[3] P. Ara, K. R. Goodearl, K. C. O'Meara and E. Pardo, Separative cancellation for projective modules over exchange rings, Israel J. Math. 105 (1998), 105-137. MR 99g:16006

[4] P. Ara, K. R. Goodearl, K. C. O'Meara and R. Raphael, $K_{1}$ of separative exchange rings and $C^{*}$-algebras with real rank zero, Pacific J. Math. 195 (2000), 261-275. MR 2001m:46155

[5] P. Ara, K. C. O'Meara and F. Perera, Stable finiteness of group rings in arbitrary characteristic, Advances in Mathematics 170 (2002), 224-238. MR 2003j:16035

[6] K. R. Goodearl, "Von Neumann Regular Rings", Pitman, London 1979; Second Ed., Krieger, Malabar, Fl., 1991. MR 93m:16006

[7] K. R. Goodearl, Von Neumann regular rings and direct sum decomposition problems, in: "Abelian groups and Modules", (Padova, 1994), Kluwer, Dordrecht, 1995, pp. 249-255.

[8] K. R. Goodearl and R. B. Warfield, JR., Algebras over zero-dimensional rings, Math. Ann. 223 (1976), 157-168. MR 54:357]

[9] M. Gromov, Asymptotic invariants of infinite groups, in: "Geometric group theory", vol. 2 (Sussex, 1991), London Math. Soc. Lecture Notes Ser. 182, Cambridge Univ. Press, Cambridge, 1993, pp. 1-295. MR 95m:20041

[10] J. Hannah and K. C. O'Meara, A new measure of growth for countable-dimensional algebras, Bull. Amer. Math. Soc. 29 (1993), 223-227. MR 94c:16035

[11] J. Hannah and K. C. O'Meara, A new measure of growth for countable-dimensional algebras. I, Trans. Amer. Math. Soc. 347 (1995), 111-136. MR 95h:16027

[12] W. K. Nicholson, Lifting idempotents and exchange rings, Trans. Amer. Math. Soc. 229 (1977), 269-278. MR 55:12757

[13] K. C. O'Meara, A new measure of growth for countable-dimensional algebras. II, J. Algebra 172 (1995), 214-240. MR 96b:16023

[14] K. C. O'Meara, The exchange property for row and column-finite matrix rings, J. Algebra 268 (2003), 744-749.

[15] E. PARDO, Metric completions of ordered groups and $K_{0}$ of exchange rings, Trans. Amer. Math. Soc. 350 (1998), 913-933. MR 98e:46088

[16] F. PererA, Lifting units modulo exchange ideals and $C^{*}$-algebras with real rank zero, $J$. Reine Angew. Math. 522 (2000), 51-62. MR 2001g:46149

[17] J. Sтоск, On rings whose projective modules have the exchange property, J. Algebra 103 (1986), 437-453. MR 88e:16038

[18] R. B. Warfield, JR., Exchange rings and decompositions of modules, Math. Ann. 199 (1972), 31-36. MR 48:11218

Departament de Matemàtiques, Universitat Autònoma de Barcelona, 08193, BelLATERra (BARCELONA), SpAin

E-mail address: para@mat.uab.es

Department of Mathematics, University of Canterbury, Christchurch, New Zealand E-mail address: K.OMeara@math. canterbury.ac.nz

Current address: Department of Mathematics, University of Connecticut, Storrs, Connecticut 06269

E-mail address: staf198@ext.canterbury.ac.nz

Department of Pure Mathematics, Queen's University Belfast, Belfast, BT7 1NN, NORTHERN IRELAND

E-mail address: perera@qub.ac.uk

Current address: Departament de Matemàtiques, Universitat Autònoma de Barcelona, 08193

Bellaterra (Barcelona), Spain

E-mail address: perera@mat.uab.es 